\title{
A Retrospective Study Evaluating Effectiveness of Concurrent And Adjuvant Temozolamide in Newly Diagnosed Glioblastoma Multiforme in Indian Population.
}

\author{
${ }^{1}$ Subbarao Nukasani, ${ }^{2}$ Kesava Ramgopal A, ${ }^{3}$ Subbarayudu S, ${ }^{4}$ Raghavendra VK, \\ ${ }^{5}$ Jagannatharaonaidu K V \\ (Department of Radiation Oncology, NRI medical college, India)
}

\begin{abstract}
The standard of care for glioblastoma is surgical resection followed by adjuvant radiotherapy with concurrent and adjuvant temozolamide. However survival data and impact of temozolamide on survival in Indian population is scanty. Between January 2014 to December 2015, patients of glioblastoma who underwent surgery and had received post-operative radiotherapy $(R T)$ with temozolomide(TMZ) were analyzed. A total of 16 patients who received RT/TMZ. Median overall survival (OS) of patients who received RT/TMZ was 11 months. On multivariate analysis age $(p=0.005), \operatorname{KPS}(p=0.05)$, extent of surgery $(p=0.043)$ were found to affect survival. Concurrent radiotherapy and temozolamide followed by adjuvant temozolamide results in improved survival in glioblastoma. The benefit of temozolomide needs to be further evaluated by larger randomized trials in Indian population with particular emphasis on MGMT promoter methylation and other molecular markers.
\end{abstract}

Keywords : Glioblastoma, radiotherapy, temozolamide

\section{INTRODUCTION}

Glioblastoma multiforme is the most frequently diagnosed primary brain tumor in adults. The standard of care includes maximal safe resection followed by radiotherapy with concurrent and adjuvant chemotherapy with temozolomide. The current standard is based on data obtained from a large multicentric randomized trial conducted across Europe and Canada[1]. However survival data and impact of temozolomide on survival in Indian population is scanty. Despite the survival advantage demonstrated by addition of temozolomide, several other prognostic factors are also known to influence outcome. Patients have thus been classified into homogenous subsets by a statistical method of recursive partitioning analysis(RPA)[2]. The prognostic value of the RTOG RPA classes has been validated[3] and applied in clinical trials. The purpose of this study is to evaluate the extent of benefit of combined modality therapy in Indian population.

\section{Materials and methods}

All patients with glioblastoma multiforme treated between January 2014 and December 2015 were included in this retrospective analysis. Patients included in the analysis had biopsy-proven glioblastoma and had received post-operative radiotherapy with or without temozolomide. Pre-operative imaging, dosimetric records and follow-up data were available for all patients included in the analysis. Patients received radiotherapy on either linear accelerator (Clinac-ix) . Radiation planning was done using 3D Conformal technique. The gross tumor volume(GTV) was defined as the enhancing tumor on T1 contrast and the T2/Flair abnormality, the clinical target volume (CTV) was defined as GTV expanded by a $1.5-2 \mathrm{~cm}$ margin. A $5 \mathrm{~mm}$ margin was given to the CTV to form the Planning target volume (PTV). Dose prescribed to PTV was 60Gy, delivered in daily fractions of $2 \mathrm{~Gy}$ over a period of 6 weeks. Chemotherapy consisting of daily temozolomide at $75 \mathrm{mg} / \mathrm{m} 2$ was administered concurrently with radiation. Additional temozolomide followed as adjuvant therapy was delivered at a dose of $150-200 \mathrm{mg} / \mathrm{m} 2$ on each of the first 5 days of every 28 -day cycle for six cycles. Patients were followed up at 1 month intervals for 6 months and 3 monthly thereafter. At each follow-up, physical examination and relevant laboratory and radiological investigations were performed. Patients were stratified according to original RTOG RPA classes (III - VI). Median overall survival was analyzed by the KaplanMeier method, with use of two-sided log-rank statistics. The Cox proportional-hazards model was fitted to adjust for stratification factors and other confounding variables.

\section{Results}

From January 2014 to December 2015, a total of 16 patients received radiotherapy and temozolomide. Median age was 50 years (range 18-76, 12 male, 04 female), 4 (25\%) patients underwent gross total resection, 6(37.5\%) had partial resection and $6(37.5 \%)$ biopsy. Median follow up period of all patients was 18 months. Median overall survival was 11 months. Median OS for RPA classes III, IV and V/VI was 20, 8 and 5.5 months and corresponding $1 \mathrm{yr}$ survival rates were $66.6 \%, 35 \%, 30 \%$ for RPA classes III, IV and V/VI 
respectively. On multivariate analysis age $(\mathrm{p}=0.005), \operatorname{KPS}(\mathrm{p}=0.05)$, extent of surgery $(\mathrm{p}=0.043)$ were found to affect survival. The survival benefit provided by addition of temozolomide was found to be greatest in RPA class III with median overall survival of 20months, survival benefit in class IV was lesser, with median overall survival of 8months. However patients belonging to class V/VI did not demonstrate significant survival benefit with the addition of temozolomide with median OS of 5.5 months.

\section{Discussion}

The advent of temozolomide in treatment of glioblastoma multiforme has created significant impact on survival as per the results of the EORTC/NCIC trial [1] conducted across Europe and Canada. Overall survival was 14.6 months in the RT/TMZ arm and 12 months in the RT only arm [1,6]. Experience with temozolomide in Indian population has been reported by Jalali et al [4]. Overall survival in 42 newly diagnosed glioblastoma patients, treated with radiotherapy and temozolomide was reported to be 16.4 months. In our institute, we report a median overall survival of 11 months in patients treated with RT/TMZ. The lower overall survival in comparison to trial results can possibly be explained by larger proportion of patients in RPA classes V/VI , which benefit minimally by addition of chemotherapy. Overall survival of patients in RPA class III was 20 months in our study which is comparable to western data. However, survival in classes IV and V,VI was 8 and 5.5 months respectively in RT/TMZ arm which is nearly half of that reported in trial data. Among the factors which positively influence survival in glioblastoma, epigenetic silencing of DNA repair gene MGMT (O6 methyl guanine DNA methyltransferase) by promoter methylation plays a prominent role [5]. In the EORTC/NCIC trial, MGMT promoter methylation was found in $45 \%$ of 206 evaluable cases which showed significantly improved overall survival of 21.7 months when treated with RT/TMZ. In contrast, the cohort which lacked the promoter methylation, failed to demonstrate improved survival on addition of temozolomide. There is minimal data regarding variations in frequency of MGMT promoter methylation in different geographical areas. With regard to Indian population, a study by Jha et al revealed a $56.8 \%$ positivity in adult glioblastoma patients evaluated by methylation specific PCR (Polymerase Chain Reaction)[7]. However there is no indian data correlating MGMT status with treatment outcome. In our study, performance of MGMT promoter methylation status could have possibly shed some light on poorer survival rates observed in RPA classes IV and V/VI. In a developing country like India, the potential role of MGMT and RPA classification in selecting patients who are likely to benefit from temozolomide cannot be over emphasized.

\section{Conclusion}

Concurrent radiotherapy and temozolomide followed by adjuvant temozolomide results in improved survival in glioblastoma. The benefit of temozolomide needs to be further evaluated by larger randomized trials in Indian population with particular emphasis on MGMT promoter methylation and other molecular markers.

\section{References}

[1]. Stupp R, Mason WP, Van Den Bent MJ, Weller M, Fisher B, Taphoorn MJ, Belanger K, Brandes AA, Marosi C, Bogdahn U, Curschmann J. Radiotherapy plus concomitant and adjuvant temozolomide for glioblastoma. New England Journal of Medicine. 2005 Mar 10;352(10):987-96.

[2]. Curran Jr WJ, Scott CB, Horton J, Nelson JS, Weinstein AS, Fischbach AJ, Chang CH, Rotman M, Asbell SO, Krisch RE, Nelson DF. Recursive partitioning analysis of prognostic factors in three Radiation Therapy Oncology Group malignant glioma trials. JNCI: Journal of the National Cancer Institute. 1993 May 5;85(9):704-10.

[3]. Mirimanoff RO, Gorlia T, Mason W, Van den Bent MJ, Kortmann RD, Fisher B, Reni M, Brandes AA, Curschmann J, Villa S, Cairncross G. Radiotherapy and temozolomide for newly diagnosed glioblastoma: recursive partitioning analysis of the EORTC 26981/22981-NCIC CE3 phase III randomized trial. Journal of Clinical Oncology. 2006 Jun 1;24(16):2563-9.

[4]. Jalali R, Basu A, Gupta T, Munshi A, Menon H, Sarin R, Goel A. Encouraging experience of concomitant Temozolomide with radiotherapy followed by adjuvant Temozolomide in newly diagnosed glioblastoma multiforme: single institution experience. British journal of neurosurgery. 2007 Jan 1;21(6):583-7.

[5]. Hegi ME,. MGMT gene silencing and benefit from temozolomide in glioblastoma. N Engl J Med 2005;352:997-1003.

[6]. Stupp R, Hegi ME, Mason WP, van den Bent MJ, Taphoorn MJ, Janzer RC, Ludwin SK, Allgeier A, Fisher B, Belanger K, Hau P. Effects of radiotherapy with concomitant and adjuvant temozolomide versus radiotherapy alone on survival in glioblastoma in a randomised phase III study: 5-year analysis of the EORTC-NCIC trial. The lancet oncology. 2009 May 31;10(5):459-66.

[7]. Jha P, Suri V, Jain A, Sharma MC, Pathak P, Jha P, Srivastava A, Suri A, Gupta D, Chosdol K, Chattopadhyay P. O6methylguanine DNA methyltransferase gene promoter methylation status in gliomas and its correlation with other molecular alterations: first Indian report with review of challenges for use in customized treatment. Neurosurgery. 2010 Dec 1;67(6):1681-91. 